\title{
Clinical utility and patient consideration in the use of lenalidomide for multiple myeloma in Chinese patients
}

This article was published in the following Dove Press journal:

OncoTargets and Therapy

2 June 2015

Number of times this article has been viewed

\section{Jing Wang \\ Hongfeng Guo \\ Xin Zhou}

Department of Hematology, Wuxi People's Hospital, Nanjing Medical University, Wuxi, People's Republic of China
Correspondence: Xin Zhou Department of Hematology, Wuxi People's Hospital, Nanjing Medical University, 299 Qingyang Road, Wuxi 21 4023, People's Republic of China $\mathrm{Tel}+8651085350201$

Email zx89232@hotmail.com

\begin{abstract}
Multiple myeloma (MM) is an incurable hematologic malignancy caused by the autonomous growth of malignant plasma cells. In the last decade, the introduction of novel targeted agents such as thalidomide, bortezomib, and lenalidomide has dramatically improved the clinical outcome of MM patients in both the frontline and recurrent settings. Lenalidomide is a synthetic derivative of thalidomide, which has been shown to significantly improve overall survival, time to progression, and overall response rates in patients with MM. The China Food and Drug Administration approved the use of lenalidomide in patients with MM in 2013. In a Phase II trial, lenalidomide plus low-dose dexamethasone was associated with a high response rate and acceptable safety profile in heavily pretreated Chinese patients with relapsed/refractory MM, including those with renal impairment and IgD subtype. However, lenalidomide will remain as a second-line antimyeloma drug in the near future because of its high price and the policy of health insurance reimbursement in People's Republic of China. In this review, we summarize the clinical utility and patient considerations in the use of lenalidomide for MM in Chinese patients. Further studies with larger sample sizes are required to investigate the better quality, longer duration, and more clinically meaningful outcomes of lenalidomide in the treatment of MM in Chinese patients.
\end{abstract}

Keywords: lenalidomide, multiple myeloma, clinical efficacy, Chinese patients

\section{Introduction}

Multiple myeloma (MM) is a neoplastic plasma cell disorder characterized by clonal proliferation of plasma cells in the bone marrow microenvironment and associated organ damage (hypercalcemia, renal insufficiency, anemia, skeletal lesions). ${ }^{1}$ The incidence of MM (age-standardized) has been estimated to be 3.5-4.5, 2.5-3.5, and 0.5-2.0 per 100,000 individuals per year in North America, Europe, and Asia (except Israel), respectively. ${ }^{2}$ It is the third most common hematologic malignancy in People's Republic of China after leukemia and non-Hodgkin's lymphoma. The annual incidence of MM in People's Republic of China is less than one per 100,000 people. ${ }^{3}$ The median survival of patients with MM was approximately 3 years before the advent of new therapies. ${ }^{4}$ The introduction of innovative therapies, such as proteasome inhibitors and immunomodulatory drugs, has dramatically improved the overall survival in MM worldwide. ${ }^{5}$ However, nearly all patients who receive these drugs or other chemotherapy will still eventually relapse and/or become subsequently resistant to treatment. Many patients with advanced relapsed/refractory MM (RRMM) in People's Republic of China have already failed on thalidomide and/or bortezomib treatments and are in need of additional effective regimens to prevent further disease progression. 
Lenalidomide (trade name: Revlimid), one of the novel drug agents used to treat $\mathrm{MM}$, is the second generation of oral immunomodulatory drugs developed by Celgene Corporation. It is a more potent molecular analog of thalidomide, which inhibits tumor angiogenesis, tumor-secreted cytokines, and tumor proliferation through the induction of apoptosis. ${ }^{6,7}$ Lenalidomide was approved by the US Food and Drug Administration (FDA) in June 2006 in combination with high-dose dexamethasone for $\mathrm{RRMM}^{8}$ and by the European Medicines Agency (EMA) in 2008. ${ }^{9}$ Lenalidomide therapy significantly improves response rates and increases progression-free survival in patients with newly diagnosed $\mathrm{MM}$ and those receiving previous antimyleoma therapy. ${ }^{10}$ In 2013, lenalidomide was recommended for the treatment of RRMM patients in the Chinese Clinical Practice Guidelines in Oncology: Multiple Myeloma. ${ }^{11}$ The approval of lenalidomide in People's Republic of China therefore expands the therapy options for Chinese patients with RRMM.

Due to the short duration of the treatment with lenalidomide applied to the Chinese patients, the effectiveness and tolerance of this drug still need to be further verified by large sample data. In this review, we summarize the clinical utility and patient considerations in the use of lenalidomide for MM in Chinese patients.

\section{Pharmacokinetics of lenalidomide}

In both healthy subjects and patients with normal renal function, the pharmacokinetics (PKs) of lenalidomide are linear and plasma exposure increases in direct proportion to the administered dose. ${ }^{12,13}$ There is no evidence of drug accumulation following multiple dosing, although the exposure to lenalidomide in MM patients increased by $57 \%$ compared with healthy volunteers. ${ }^{12,13}$ Lenalidomide is quickly absorbed after oral administration; maximum plasma concentrations $\left(\mathrm{C}_{\max }\right)$ occur $0.6-1.5$ hours and 0.5-4.0 hours post-dose in healthy male volunteers and $\mathrm{MM}$ patients, respectively. ${ }^{12,13}$ Coadministration with a high-fat meal reduced $\mathrm{C}_{\max }$ by approximately $50 \%$, and delayed time to $\mathrm{C}_{\max }$ by 1.63 hours. However, Phase III trials were dosed without regard to food; therefore, clinical relevance of the food effect was minimal. ${ }^{14}$

Lenalidomide undergoes hydrolysis in human plasma, with two metabolites in both blood and urine, 5-OH-lenalidomide and $\mathrm{N}$-acetyl-lenalidomide. Lenalidomide is primarily eliminated as unchanged drug with urinary excretion (90\%) and in minor quantities with feces $(4 \%) .{ }^{15}$ The metabolism contributes to a very minor extent to the clearance of lenalidomide in humans. The mean terminal elimination half-life $\left(\mathrm{t}_{1 / 2}\right)$ was approximately $3-4$ hours in clinically relevant dose ranges. ${ }^{15}$ Renal impairment reduces the clearance, increases plasma exposure, and prolongs the $t_{1 / 2}$ of lenalidomide, although oral absorption, protein binding, and nonrenal elimination are unaltered..$^{16}$ As renal impairment progressed to moderate, severe, or end-stage renal disease, total and renal lenalidomide clearance decreased drastically, and $\mathrm{t}_{1 / 2}$ was prolonged by approximately $6-12$ hours and increasing plasma exposure by two- to fourfold. ${ }^{16}$ Therefore, adjustments to the starting dose of lenalidomide are recommended for all MM patients with moderate or severe renal insufficiency (creatinine clearance $<60 \mathrm{~mL} / \mathrm{min}$ ) to maintain effective and safe exposure levels. ${ }^{17}$ Lenalidomide does not show clinically relevant PK drug-drug interaction when coadministered with cytochrome P450 inhibitors, because it is not metabolized extensively by the $\mathrm{P} 450$ liver enzymes. ${ }^{15}$

The PK profile of lenalidomide in Chinese patients was similar to that historically reported in North American and Japanese patients with RRMM and normal renal function (Table 1). ${ }^{12,13,18,19}$ There were no apparent differences in any of the multiple-dose PK parameters for lenalidomide alone or lenalidomide plus dexamethasone. The addition of dexamethasone had no effect on the plasma exposure to lenalidomide.

\section{Mechanisms of action of lenalidomide}

The exact mechanisms of lenalidomide are not yet clearly defined, but studies have shown that lenalidomide may work through various biological effects in MM. Lenalidomide can rapidly reduce the MM tumor burden and sustain disease control through its unique mechanism of action, including both tumoricidal and immunomodulatory effects. ${ }^{20}$

Lenalidomide has been shown to modulate different components of the immune system by altering cytokine production, regulating T-cell costimulation and augmenting the NK cell cytotoxicity. ${ }^{7}$ This drug can inhibit the secretion of inflammatory factors like TNF- $\alpha$, IL-1, IL-6, and IL-12 and promote the human peripheral blood mononuclear cells to secrete anti-inflammatory cytokine IL-10, thus inhibit the proliferation of myeloma cells and induce the apoptosis of the tumor cells. ${ }^{21}$ It acts on T-cells through the B7-CD28 costimulatory signaling pathway to promote Th1 cells to secret interferon and IL-2, then promote the proliferation of clonal T-cells and activation of NK cells. ${ }^{22}$ Lenalidomide also could enhance the antibody-dependent cell-mediated cytotoxicity and natural cytotoxicity of NK cells, ${ }^{23}$ thus it plays a role in antitumor effects. In addition to inducing cell-cycle arrest, lenalidomide could enhance the effect of TNF-related apoptosis-inducing ligand (TRAIL), inhibit 
Table I Comparison of plasma lenalidomide PK parameters (administered with dexamethasone) in different populations of relapsed myeloma patients who had normal renal function (creatinine clearance $\geq 60 \mathrm{~mL} / \mathrm{min}$ )

\begin{tabular}{|c|c|c|c|}
\hline PK parameter & $\begin{array}{l}\text { Caucasian MM } \\
\text { patients }(n=34)\end{array}$ & $\begin{array}{l}\text { Japanese MM } \\
\text { patients }(n=\mid 2)\end{array}$ & $\begin{array}{l}\text { Chinese MM } \\
\text { patients }(n=9)\end{array}$ \\
\hline $\mathrm{C}_{\max }(\mathrm{ng} / \mathrm{mL})$ & $487(35.0)$ & $572(33.2)$ & $596(30.2)$ \\
\hline $\mathrm{T}_{\max }(\mathrm{h})$ & $1.0(0.4-4.0)$ & $1.0(0.4-2.0)$ & $0.93(0.5-1.0)$ \\
\hline $\mathrm{AUC}_{\infty}(\mathrm{h} \cdot \mathrm{ng} / \mathrm{mL})$ & $2,124(28.6)$ & $2,305(23.7)$ & $2,202(30.6)$ \\
\hline $\mathrm{CL} / \mathrm{F}(\mathrm{mL} / \mathrm{min})$ & $196(28.7)$ & I8I (23.7) & I84 (30.7) \\
\hline$t_{1 / 2}(h)$ & $3.18(20.7)$ & $2.70(19.3)$ & $3.18(39.0)$ \\
\hline $\mathrm{Vz} / \mathrm{F}(\mathrm{L})$ & $54.0(29.5)$ & $41.8(14.3)$ & $50.7(28.4)$ \\
\hline
\end{tabular}

Note: a Geometric mean (geometric coefficient of variation \%) data are presented for all parameters except when stated as median (range). Reproduced with permission from Hou J, Du X, Jin J, et al. A multicenter, open-label, phase 2 study of lenalidomide plus low-dose dexamethasone in Chinese patients with relapsed/refractory multiple myeloma: the MM-02I trial. J Hematol Oncol. 201 3;6:4I. ${ }^{19}$

Abbreviations: $A \cup C_{\infty}$, area under the plasma concentration-time curve from time 0 to infinity; $C L / F$, apparent total plasma clearance; $C_{\text {max }}$, maximum plasma concentration; MM, multiple myeloma; PK, pharmacokinetic; $t_{1 / 2}$, terminal elimination half-life; $T_{\max }$, time to $C_{\max } ; V_{z} / F$, apparent volume of distribution during terminal phase after nonintravenous administration.

apoptin-2, increase Fas-mediated cell death, and upregulate the activity of cysteine-aspartic acid protease (caspase-8), downregulate the expression of caspase- 8 inhibitor (FLIP, Ciap2), downregulate the activity of NF- $\kappa \mathrm{B}$, and inhibit the activity of insulin-like growth factor (IGF-1) and thereby exert a direct antitumor effect. ${ }^{24}$

Lenalidomide could inhibit angiogenesis by decreasing the expression of angiogenic factors, such as vascular endothelial growth factor (VEGF) and fibroblast growth factors in MM. ${ }^{25}$ Furthermore, lenalidomide acts on bone marrow microenvironment, which could directly inhibit the growth of tartrate-resistant acid phosphate (TRAP) positive osteoclasts. ${ }^{26}$ It could also downregulate the expression level of cell adhesion molecules such as ICAM-1, VCAM-1, and E-selectin, thus inhibit the adhesion of MM cells and BMSC. ${ }^{27}$ This may lead to disruption of stromal support and have subsequent effects on MM cell migration, growth, and survival.

In a study by Gandhi et al lenalidomide in combination with dexamethasone synergistically upregulated tumor suppressor genes and caspases, resulting in increased cell-cycle arrest and a higher rate of MM cell apoptosis. ${ }^{28}$ However, the addition of dexamethasone also antagonized the immunomodulatory effects of lenalidomide in a dose-dependent manner. ${ }^{28}$

The precise molecular target of lenalidomide has been difficult to quantify until recently, when thalidomide was shown to physically interact with (and hence inhibit) the E3 ubiquitin ligase cereblon (CRBN) ${ }^{29}$ The article by Krönke et $\mathrm{al}^{30}$ demonstrates that lenalidomide causes selective ubiquitination and degradation of two lymphoid transcription factors, Ikaros family zinc finger proteins 1 and 3 (IKZF1 and IKZF3), by the CRBN-CRL4 ubiquitin ligase. An article by Lu et $\mathrm{al}^{31}$ supports these observations. These findings reveal a novel mechanism of action for lenalidomide: alteration of the activity of an E3 ubiquitin ligase, leading to selective degradation of specific targets. CRBN has been considered as one of the immunomodulatory drugs' direct target proteins. It may provide a biomarker to predict immunomodulatory drug response and resistance as well as facilitate the development of new drugs with more specific antimyeloma activities.

\section{Chinese efficacy studies of lenalidomide}

Lenalidomide was approved for the treatment of adult patients with MM who have received at least one prior chemotherapy by the China FDA in January 2013. MM-02 $1^{19}$ is a multicenter, open-label, Phase II clinical trial about lenalidomide in combination with low-dose dexamethasone $(\mathrm{Rd})$ to treat Chinese RRMM patients. In this study, lenalidomide $25 \mathrm{mg}$ was given orally once daily on days 1-21 of each 28-day cycle and dexamethasone $40 \mathrm{mg}$ once daily on days 1,8 , 15 , and 22 of each 28-day cycle. The results showed, in the efficacy-evaluable population $(n=187)$, the overall response rate (ORR) was $47.6 \%$, including 23 patients (12.3\%) with a very good partial response and seven patients (3.7\%) who had a complete response. An additional 88 patients (47.1\%) achieved stable disease, resulting in a disease control rate (stable disease or better) of $94.7 \%$. Patients with renal impairment and the immunoglobulin $\mathrm{D}(\mathrm{IgD})$ disease subtype also had clinically meaningful response rates.

In contrast to patients in the MM-009 and MM-010 studies, patients in MM-021 had advanced disease (85.6\% had Durie-Salmon MM stage III) and were more heavily pretreated (56.7\% had received four or more prior regimens), and more patients were previously exposed to bortezomib $(63.1 \%)$, thalidomide $(69.5 \%)$, or both $(44.9 \%)$. In addition, $5.4 \%$ of patients had the IgD subtype of MM. The ORR in this series was slightly lower than the results in the MM-009 
and MM-010 studies and Spanish and Greek retrospective studies, but was consistent with the result from the Korean patients (Table 2), ${ }^{32-37}$ perhaps explained by dexamethasone dosing and disease severity differences. Of note, MM-021 patients who had received two or fewer treatments prior to $\mathrm{Rd}$ had a higher ORR (58\%) than the overall efficacy population - closer to the rate reported in the MM-009 and MM-010 studies. ${ }^{19}$ These findings suggest that Rd treatment may be a significant improvement in benefit when used at first relapse compared with its use later as salvage therapy, which is consistent with a published result. ${ }^{38}$

After a median study follow-up of 15.2 months in MM-021, the median duration of response was 8.8 months, and median progression-free survival was 8.3 months. Moreover, the median progression-free survival in MM-021 is consistent with observations of time to progression observed in the MM-009 and MM-010 studies, in which the median times to progression were 11.1 and 11.3 months, respectively. ${ }^{32,33}$

\section{Safety and tolerability of lenalidomide in Chinese patients}

In the MM-021 trial, Rd was associated with an acceptable safety profile in heavily pretreated Chinese patients with RRMM, including those with renal impairment and IgD subtype. The most frequently reported adverse events (AEs) of lenalidomide were related to blood system disorders, gastrointestinal disorders, general disorders, skin and subcutaneous tissue disorders, and administrative site conditions. Similar to other studies (Table 3), ${ }^{32-37}$ hematologic toxicities such as anemia, neutropenia, and thrombocytopenia were most common in MM-021 and were manageable by dose adjustment of lenalidomide. In the MM-009 and MM-010 studies, the median number of previous treatment lines was two; prior exposure to thalidomide was lower than in our study at $41.8 \%$ and $30.1 \%$, and prior exposure to bortezomib was $10.7 \%$ and $4.5 \%$, respectively, for the lenalidomide-treated groups. In contrast to patients in the MM-009 and MM-010 studies, patients in this series were more heavily pretreated (median number of prior treatment lines was four), and more patients were previously exposed to bortezomib (63.1\%), thalidomide $(69.5 \%)$, or both $(44.9 \%)$. It is interesting to note that, despite being a more heavily pretreated group, those with two or more prior therapies generally did not experience more treatment-emergent AEs.

In MM-021, the most common grade 3-4 AEs were anemia $(26.1 \%)$, neutropenia $(25.1 \%)$, thrombocytopenia (14.6\%), pneumonia (13.1\%), leukopenia (9.5\%), and decreased neutrophil count $(8.5 \%)$. AEs resulted in dose reduction or interruption of lenalidomide in 80 patients $(40.2 \%)$ and of dexamethasone in 87 patients $(43.7 \%)$. Treatment-emergent AEs resulted in discontinuation of lenalidomide treatment in 18 patients (9\%). Cardiac failure, thrombocytopenia, pneumonia, and constipation led to discontinuation of lenalidomide in two patients each. The incidence of grade 3-4 cytopenias and pneumonia were consistent with safety findings from the MM-009 and MM-010 studies. But the incidence of grade 3-4 anemia was higher in MM-021 than in the global pivotal Phase III studies (26.1\% versus $13 \%$ and $8.6 \%$ ), which may be a result of the already high baseline incidence of anemia (87\% in MM-021), partly related to underlying disease characteristics. ${ }^{19,32,33}$ Neutropenia and thrombocytopenia were managed with appropriate dose reduction of lenalidomide and/or the administration of granulocyte colonystimulating factor and platelet transfusion. The increased risk of infection with lenalidomide treatment suggests that antibiotic prophylaxis should be considered as part of the

Table 2 Response of lenalidomide plus dexamethasone treatment in patients with relapsed/refractory multiple myeloma

\begin{tabular}{|c|c|c|c|c|c|c|c|c|c|c|c|}
\hline Study & Location & $\begin{array}{l}\text { Number of } \\
\text { patients }^{\mathrm{a}}\end{array}$ & Regimen & $\begin{array}{l}\text { ORR } \\
(\%)\end{array}$ & CR & VGPR & PR & $\begin{array}{l}\text { TTP } \\
\text { (months) }\end{array}$ & $\begin{array}{l}\text { TTR } \\
\text { (months) }\end{array}$ & $\begin{array}{l}\text { PFS } \\
\text { (months) }\end{array}$ & $\begin{array}{l}\text { DOR } \\
\text { (months) }\end{array}$ \\
\hline MM-00933 & USA and Canada & 177 & RD & 61 & 14.1 & 10.2 & 36.7 & 11.1 & NA & NA & 15.8 \\
\hline$M M-010^{32}$ & $\begin{array}{l}\text { Europe, Israel, } \\
\text { Australia }\end{array}$ & 176 & RD & 60.2 & 15.9 & 8.5 & 35.8 & NA & NA & NA & 16.5 \\
\hline MM-0I835 & Spain & 63 & RD & 78 & 21 & 21 & 37 & 13.3 & 2.8 & 13.3 & 18.4 \\
\hline Katodritou et $\mathrm{al}^{36}$ & Greece & 212 & RD & 77.4 & 20.2 & NA & NA & NA & 2.0 & NA & 34.4 \\
\hline$M M-02 I^{19}$ & $\begin{array}{l}\text { People's Republic } \\
\text { of China }\end{array}$ & 187 & Rd & 47.6 & 3.7 & 12.3 & 43.9 & NA & 1.9 & 8.3 & 8.8 \\
\hline Kim et $\mathrm{al}^{37}$ & Korea & 110 & Rd & 43.7 & 1.8 & 13.7 & 28.2 & 8.0 & NA & NA & NA \\
\hline lida et $\mathrm{al}^{18}$ & Japan & 6 & RD & 100 & 33.3 & NA & NA & NA & NA & NA & NA \\
\hline
\end{tabular}

Notes: ${ }^{2}$ Those who received lenalidomide in combination with dexamethasone. RD: lenalidomide 25 mg was given orally once daily on days I-2I of each 28 -day cycle; dexamethasone $40 \mathrm{mg}$ once daily on days I-4, 9-12, and I7-20 for the first four cycles, then only on days I-4 after the fourth cycle. Rd: lenalidomide 25 mg was given orally once daily on days I-2I of each 28-day cycle; dexamethasone $40 \mathrm{mg}$ once daily on days I, 8, I5, and 22 of each 28-day cycle.

Abbreviations: CR, complete response; DOR, duration of response; NA, not available; ORR, overall response rate; PFS, progression-free survival; PR, partial response; $\mathrm{RD}$, lenalidomide in combination with low-dose dexamethasone; Rd, lenalidomide in combination with high-dose dexamethasone; TTP, time to progression; TTR, time to relapse; VGPR, very good partial response. 
Table 3 Grade 3-4 treatment-emergent AEs reported on lenalidomide and dexamethasone

\begin{tabular}{|c|c|c|c|c|c|c|}
\hline & MM-00933 & $M M-010^{32}$ & $M M-018^{35}$ & MM-02II9 & Kim et $\mathrm{al}^{37}$ & lida et $\mathrm{al}^{18}$ \\
\hline \multicolumn{7}{|l|}{ Hematologic AEs (\%) } \\
\hline Neutropenia & 41.2 & 29.5 & 51 & 25.1 & 59.1 & 66.7 \\
\hline Thrombocytopenia & 14.2 & 11.4 & 14 & 14.6 & 43.6 & NA \\
\hline Anemia & 13 & 8.6 & 11 & 26.1 & 43.6 & 13.3 \\
\hline \multicolumn{7}{|l|}{ Nonhematologic AEs (\%) } \\
\hline Constipation & 2.8 & 1.7 & NA & NA & 17.2 & NA \\
\hline Diarrhea & 3.4 & 2.8 & NA & NA & NA & NA \\
\hline Upper respiratory infection & I.I & 1.7 & NA & 4.0 & NA & NA \\
\hline Pneumonia & 12.4 & NA & NA & 18.6 & 3.5 & 6.2 \\
\hline Deep-vein thrombosis & NA & 4.0 & 6 & NA & 2.7 & 0 \\
\hline Fatigue & 6.2 & 6.8 & NA & 4.0 & NA & NA \\
\hline Hypokalemia & 6.2 & NA & NA & 7.0 & NA & NA \\
\hline Hyperglycemia & 10.8 & NA & NA & 2.5 & NA & NA \\
\hline Discontinuation due to AEs (\%) & NA & 19.8 & 17 & 9 & NA & NA \\
\hline
\end{tabular}

Abbreviations: AEs, adverse events; NA, not available.

treatment regimen. ${ }^{33,35,36}$ However, prophylactic antibiotics did not be administered on lenalidomide treatment for MM patients in other studies. ${ }^{19,32,37}$ More studies on this issue are still required in the future.

Use of lenalidomide as monotherapy is not associated with an increased risk of developing venous thromboembolic. ${ }^{39}$ However, when lenalidomide is combined with dexamethasone, the incidence rate of grade 3 or higher venous thromboembolic increases greatly in RRMM patients without preventive measures. ${ }^{32,33}$ Different from Western data, including the MM-009, MM-010, and MM-018 studies, the incidence of thromboembolic events in the Chinese series was lower $(14.7 \%, 11.4 \%$, and $14 \%$ versus $0.5 \%) .{ }^{19,32,33,35}$ This may be due to undergoing antithrombotic prophylactic treatment in all patients and/or the overall shorter time on study for patients in MM-021. In addition, low incidence of thromboembolism has been described in published series in Asian populations compared with Caucasian populations, including among patients with cancer. ${ }^{18,37,40,41}$ Thromboprophylaxis is indicated for patients being treated with lenalidomide to reduce the risk of thromboembolic events to a level equivalent to the background risk in myeloma patients. ${ }^{42}$

The study of MM-021 revealed an incidence of grade 3-4 fatigue (4.0\%), hypokalemia (7.0\%), and hyperglycemia (2.5\%), which was similar to the MM-009 trial. There was also a low incidence of grade 3-4 sedation, fatigue, and rash. For those patients with one or two or more prior therapies, the incidence of treatment-emergent peripheral neuropathy (PN) was low, and no grade 3-4 PN was reported. Importantly, lenalidomide was not associated with PN in this study. Recent attention has been given to the potential impact of long-term lenalidomide treatment on the incidence of second primary malignancies (SPMs) in newly diagnosed patients with MM. ${ }^{43}$ SPM was not evaluated in detail in MM-021, but a patient developed an SPM that was not considered to be related to either lenalidomide or dexamethasone during a median 15.2-month follow-up period. The most common cause of death in MM-021 was MM or complications due to disease progression, which is consistent with what is expected for a population of patients with RRMM.

\section{Cost-effectiveness}

Estimating the costs and outcomes of alternative treatment strategies for patients with RRMM is important in order to ensure that patients receive effective and efficient therapy from the limited health care resources available. The information about the cost-effectiveness of lenalidomide is still lacking. When costs are considered in the health economic analyses of lenalidomide, these are based on simulation modeling, rather than the direct collection of cost and outcome data from trials.

Recent studies using the same discrete-event simulation showed that combination therapy with lenalidomidedexamethasone (RD) may be associated with a higher cost, but may also be associated with greater effectiveness compared with its main comparator for patients with RRMM in some countries. ${ }^{44}{ }^{47} \mathrm{RD}$ therapy is cost-effective in second-line RRMM patients with 3.2 life-years (LYs) and 2.2 qualityadjusted LYs (QALY), and such patients were found to have an incremental cost- effectiveness ratio (ICER) of $£ 30,153$ / QALY versus dexamethasone alone. ${ }^{44}$ The study conducted from the perspective of Norway further reported that RD resulted in a greater gain in LYs and QALYs compared with bortezomib plus dexamethasone (4.06 LYs and 2.95 QALYs versus 3.11 LYs and 2.19 QALYs, respectively). Costs in 
Norway were also higher for RD compared with bortezomib plus dexamethasone, with total lifetime costs in 2010 per patient of US $\$ 114,868$ or $€ 86,151$ and US\$83,493 or $€ 62,620$, respectively. ${ }^{45} \mathrm{RD}$ has meaningful cost benefits compared to bortezomib from the US payer perspective. The average total cost per day was US\$47 in 2011 lower for RD versus bortezomib ( $\$ 285 /$ day and $\$ 332 /$ day, respectively). Treatment with bortezomib had annual excess total costs of $>\$ 17,000$ compared with RD. Total cost per month without progression was $11 \%$ lower with RD.

Cost-effectiveness analyses are country specific and their results are not readily transferrable to other settings because of the differing currencies, the discordant timing of the analyses, the diverse patient populations studied, and the differing drug prices. In People's Republic of China, the development of pharmacoeconomics is slow and conducting pharmacoeconomic research is difficult, ${ }^{48}$ and data on the cost of lenalidomide treatment are not available. Therefore, the cost-effectiveness analysis of lenalidomide is important for the fields of medicine and policy and deserves further investigation.

\section{Patient-focused perspectives}

Lenalidomide has an acceptable safety profile with manageable AEs. ${ }^{39}$ Importantly, its tolerability and lack of cumulative toxicity allow for continuous lenalidomide use after autologous stem cell transplantation or in relapsed disease. ${ }^{49}$ The Spanish cohort of the MM-018 study recently reported improvement in health-related quality of life and symptom scores regardless of treatment response in patients with RRMM treated with long-term lenalidomide. ${ }^{35}$ Although lenalidomide has the potential to change the way in which MM is treated, shifting perceptions from MM as an incurable acute illness to a disease that can be managed with long-term therapy, ${ }^{49}$ the high price limits the use of lenalidomide in People's Republic of China.

Not only is efficacy of therapy important, but costeffectiveness is also a critical consideration for patients and cancer treatment centers, especially in resource-limited settings such as People's Republic of China. Constrained economic conditions result in different treatment options. Clinicians must consider costs when recommending treatment regimens. People's Republic of China's medical care system is very different from that of other developed countries, in that some patients do not have medical insurance, and many medications and treatments are not covered by medical insurance, such as lenalidomide. It means patients pay out of pocket if they choose to use lenalidomide therapy. The cost of one course of lenalidomide (25 mg once daily on days 1-21 of each 28-day cycle) is 59,000 RMB ( $\$ 9,346.53 ; 1 \mathrm{USD}=$ 6.3125 RMB in 2013), which does not include the extra costs associated with use of lenalidomide. As a potential option, decreasing the price of lenalidomide or giving a discount plan for lenalidomide would improve the use of lenalidomide in People's Republic of China. In 2013, lenalidomide came into the Chinese market, and it was on the list of public benefit activities of the China International Charity Foundation. Some patients can participate in the activity of buy three get nine free, by which the total expense is 177,000 RMB $(\$ 28,039.60)$ in 1 year. However, People's Republic of China's gross domestic product per capita was $41,907.59 \mathrm{RMB}(\$ 6,638.83)$ in $2013,{ }^{50}$ which means that most patients cannot afford lenalidomide for the treatment of MM. Decreasing the price of lenalidomide is a potential option for meeting the requirements for the approval of coverage of the drug by the health insurance system in People's Republic of China.

\section{Conclusion}

Lenalidomide is an orally active immunomodulatory drug. A combination of lenalidomide and low-dose dexamethasone provided an effective and well-tolerated treatment option in heavily pretreated Chinese patients with RRMM, including those with renal impairment and $\operatorname{IgD}$ subtype. However, lenalidomide will remain as a second-line antimyeloma drug in the near future because of its high price and the policy of health insurance reimbursement in People's Republic of China. Further studies with larger sample sizes are required to investigate the long-term safety and efficacy of lenalidomide in the treatment of MM in Chinese patients.

\section{Disclosure}

The authors report no conflicts of interest in this work.

\section{References}

1. Kyle RA, Rajkumar SV. Treatment of multiple myeloma: a comprehensive review. Clin Lymphoma Myeloma. 2009;9(4):278-288.

2. Tricot G. Multiple myeloma and other plasma cell disorders. In: Hoffman R, Benz EJ Jr, Shattil SJ, et al, editors. Hematology Basic Principles and Practice. 4th Ed. Elsevier; 2005:1501-1505.

3. GLOBOCAN 2012: Estimated Cancer incidence, Mortality and Prevalence Worldwide in 2012 [webpage on the Internet]. Lyon: International Agency for Research on Cancer. Available from: http://globocan.iarc.fr/ Pages/fact_sheets_population.aspx. Accessed March 31, 2015.

4. Munshi NC, Anderson KC. New strategies in the treatment of multiple myeloma. Clin Cancer Res. 2013;19(13):3337-3344.

5. Kumar SK, Rajkumar SV, Dispenzieri A, et al. Improved survival in multiple myeloma and the impact of novel therapies. Blood. 2008;111(5): 2516-2520.

6. Hideshima T, Chauhan D, Shima Y, et al. Thalidomide and its analogs overcome drug resistance of human multiple myeloma cells to conventional therapy. Blood. 2000;96(9):2943-2950. 
7. Kotla V, Goel S, Nischal S, et al. Mechanism of action of lenalidomide in hematological malignancies. J Hematol Oncol. 2009;2:36.

8. US Food and Drug Administration (FDA) [homepage on the Internet]. Silver Spring: US Food and Drug Administration (FDA). Available from: www.fda.gov/. Accessed May 13, 2014.

9. European Medicines Agency (EMA) [homepage on the Internet]. London: EMA. Available from: www.ema.europa.eu. Accessed May 13, 2014.

10. Dimopoulos MA, Terpos E, Niesvizky R. How lenalidomide is changing the treatment of patients with multiple myeloma. Crit Rev Oncol Hematol. 2013;88 Suppl 1:S23-S35.

11. Chinese Medical Association, Chinese Society of Hematology, Multiple Myeloma Working Party of the Chinese Group. [Guidelines for treatment of multiple myeloma]. Zhonghua Nei Ke Za Zhi. 2013;52(9):791-795. Chinese.

12. Revlimid ${ }^{\circledR}$ (lenalidomide) [package insert]. Celgene Corporation.

13. Richardson PG, Schlossman RL, Weller E, et al. Immunomodulatory drug CC-5013 overcomes drug resistance and is well tolerated in patients with relapsed multiple myeloma. Blood. 2002;100(9): 3063-3067.

14. Chen N, Kasserra C, Reyes J, Liu L, Lau L. Single-dose pharmacokinetics of lenalidomide in healthy volunteers: dose proportionality, food effect, and racial sensitivity. Cancer Chemother Pharmacol. 2012;70(5):717-725.

15. Chen N, Wen L, Lau H, Surapaneni S, Kumar G. Pharmacokinetics, metabolism and excretion of [(14)C]-lenalidomide following oral administration in healthy male subjects. Cancer Chemother Pharmacol. 2012;69(3):789-797.

16. Chen N, Lau H, Kong L, et al. Pharmacokinetics of lenalidomide in subjects with various degrees of renal impairment and in subjects on hemodialysis. J Clin Pharmacol. 2007;47(12):1466-1475.

17. European Medicines Agency. Available from: http://www.ema. europa.eu/docs/enGB/documentlibrary/EPAR-ProductInformation/ human/000717/WC500056018.pdf. Accessed May 24, 2014.

18. Iida $\mathrm{S}$, Chou $\mathrm{T}$, Okamoto $\mathrm{S}$, et al. Lenalidomide plus dexamethasone treatment in Japanese patients with relapsed/refractory multiple myeloma. Int J Hematol. 2010;92(1):118-126.

19. Hou J, Du X, Jin J, et al. A multicenter, open-label, phase 2 study of lenalidomide plus low-dose dexamethasone in Chinese patients with relapsed/refractory multiple myeloma: the MM-021 trial. J Hematol Oncol. 2013;6:41.

20. Chang X, Zhu Y, Shi C, Stewart AK. Mechanism of immunomodulatory drugs' action in the treatment of multiple myeloma. Acta Biochim Biophys Sin (Shanghai). 2014;46(3):240-253.

21. Corral LG, Haslett PA, Muller GW, et al. Differential cytokine modulation and $\mathrm{T}$ cell activation by two distinct classes of thalidomide analogues that are potent inhibitors of TNF-alpha. J Immunol. 1999;163(1): 380-386.

22. LeBlanc R, Hideshima T, Catley LP, et al. Immunomodulatory drug costimulates T cells via the B7-CD28 pathway. Blood. 2004;103(5): $1787-1790$.

23. Hayashi T, Hideshima T, Akiyama M, et al. Molecular mechanisms whereby immunomodulatory drugs activate natural killer cells: clinical application. Br J Haematol. 2005;128(2):192-203.

24. Mitsiades N, Mitsiades CS, Poulaki V, et al. Apoptotic signaling induced by immunomodulatory thalidomide analogs in human multiple myeloma cells: therapeutic implications. Blood. 2002;99(12):4525-4530.

25. Gupta D, Treon SP, Shima Y, et al. Adherence of multiple myeloma cells to bone marrow stromal cells upregulates vascular endothelial growth factor secretion: therapeutic applications. Leukemia. 2001;15(12):1950-1961.

26. Breitkreutz I, Raab MS, Vallet S, et al. Lenalidomide inhibits osteoclastogenesis, survival factors and bone-remodeling markers in multiple myeloma. Leukemia. 2008;22(10):1925-1932.

27. Geitz H, Handt S, Zwingenberger K. Thalidomide selectively modulates the density of cell surface molecules involved in the adhesion cascade. Immunopharmacology. 1996;31(2-3):213-221.
28. Gandhi AK, Kang J, Capone L, et al. Dexamethasone synergizes with lenalidomide to inhibit multiple myeloma tumor growth, but reduces lenalidomide-induced immunomodulation of $\mathrm{T}$ and $\mathrm{NK}$ cell function. Curr Cancer Drug Targets. 2010;10(2):155-167.

29. Ito T, Ando H, Suzuki T, et al. Identification of a primary target of thalidomide teratogenicity. Science. 2010;327(5971):1345-1350.

30. Krönke J, Udeshi ND, Narla A, et al. Lenalidomide causes selective degradation of IKZF1 and IKZF3 in multiple myeloma cells. Science. 2014;343(6168):301-305

31. Lu G, Middleton RE, Sun H, et al. The myeloma drug lenalidomide promotes the cereblon-dependent destruction of Ikaros proteins. Science. 2014;343(6168):305-309.

32. Dimopoulos M, Spencer A, Attal M, et al; Multiple Myeloma (010) Study Investigators. Lenalidomide plus dexamethasone for relapsed or refractory multiple myeloma. N Engl J Med. 2007;357(21):2123-2132.

33. Weber DM, Chen C, Niesvizky R, et al; Multiple Myeloma (009) Study Investigators. Lenalidomide plus dexamethasone for relapsed multiple myeloma in North America. $N$ Engl $J$ Med. 2007;357(21):2133-2142.

34. Dimopoulos MA, Chen C, Spencer A, et al. Long-term follow-up on overall survival from the MM-009 and MM-010 phase III trials of lenalidomide plus dexamethasone in patients with relapsed or refractory multiple myeloma. Leukemia. 2009;23(11):2147-2152.

35. Alegre A, Oriol-Rocafiguera O, Garcia-Larana J, et al. Efficacy, safety and quality-of-life associated with lenalidomide plus dexamethasone for the treatment of relapsed or refractory multiple myeloma: the Spanish experience. Leuk Lymphoma. 2012;53(9):1714-1721.

36. Katodritou K, Vadikolia C, Lalagianni C, et al. "Real-world" data on the efficacy and safety of lenalidomide and dexamethasone in patients with relapsed/refractory multiple myeloma who were treated according to the standard clinical practice: a study of the Greek Myeloma Study Group. Ann Hematol. 2014;93(1):129-139.

37. Kim K, Kim SJ, Voelter V, et al; Korean Multiple Myeloma Working Party (KMMWP). Lenalidomide with dexamethasone treatment for relapsed/refractory myeloma patients in Korea-experience from 110 patients. Ann Hematol. 2014;93(1):113-121.

38. Stadtmauer EA, Weber DM, Niesvizky R, et al. Lenalidomide in combination with dexamethasone at first relapse in comparison with its use as later salvage therapy in relapsed or refractory multiple myeloma. Eur J Haematol. 2009;82(6):426-432.

39. Richardson P, Jagannath S, Hussein M, et al. Safety and efficacy of single-agent lenalidomide in patients with relapsed and refractory multiple myeloma. Blood. 2009;114(4):772-778.

40. White RH, Keenan CR. Effects of race and ethnicity on the incidence of venous thromboembolism. Thromb Res. 2009;123 Suppl 4: S11-S17.

41. Chew HK, Wun T, Harvey D, Zhou H, White RH. Incidence of venous thromboembolism and its effect on survival among patients with common cancers. Arch Intern Med. 2006;166(4):458-464.

42. Palumbo A, Rajkumar SV, Dimopoulos MA, et al; International Myeloma Working Group. Prevention of thalidomide- and lenalidomide-associated thrombosis in myeloma. Leukemia. 2008;22(2): 414-423.

43. Palumbo A, Bringhen S, Kumar SK, et al. Second primary malignancies with lenalidomide therapy for newly diagnosed myeloma: a metaanalysis of individual patient data. Lancet Oncol. 2014;15(3): 333-342.

44. Brown RE, Stern S, Dhanasiri S, Schey S. Lenalidomide for multiple myeloma: cost-effectiveness in patients with one prior therapy in England and Wales. Eur J Health Econ. 2013;14(3):507-514.

45. Möller J, Nicklasson L, Murthy A. Cost-effectiveness of novel relapsed-refractory multiple myeloma therapies in Norway: lenalidomide plus dexamethasone vs bortezomib. J Med Econ. 2011;14(6): 690-697.

46. Durie B, Binder G, Pashos C, Khan Z, Hussein M, Borrello I. Total cost comparison in relapsed/refractory multiple myeloma. J Med Econ. 2013;16(5):614-622. 
47. Fragoulakis V, Kastritis E, Psaltopoulou T, Maniadakis N. Economic evaluation of therapies for patients suffering from relapsed-refractory multiple myeloma in Greece. Cancer Manag Res. 2013;5:37-48.

48. Bao Peng L, Qing Tan C, Mini Wan X, Cui W. Cost-utility analysis in China: differences and difficulties compared with developed countries. Pharmacoeconomics. 2007;25(7):619.

49. Dimopoulos MA, Terpos E. Lenalidomide: an update on evidence from clinical trials. Blood Rev. 2010;24 Suppl 1:S21-S26.
50. National Data [homepage on the Internet]. Beijing: National Bureau of Statistics of China. Available from: http://data.stats.gov.cn/workspace/ index $? \mathrm{a}=\mathrm{q} \&$ type $=\mathrm{global} \& \mathrm{db} \operatorname{code}=\mathrm{hgnd} \& \mathrm{~m}=$ hgnd $\&$ dimension $=\mathrm{zb} \& \mathrm{co}$ $\mathrm{de}=\mathrm{A} 020108 \&$ region $=000000 \&$ time $=2013,2013$. Accessed October 8 , 2014.

\section{Publish your work in this journal}

OncoTargets and Therapy is an international, peer-reviewed, open access journal focusing on the pathological basis of all cancers, potential targets for therapy and treatment protocols employed to improve the management of cancer patients. The journal also focuses on the impact of management programs and new therapeutic agents and protocols on

patient perspectives such as quality of life, adherence and satisfaction. The manuscript management system is completely online and includes a very quick and fair peer-review system, which is all easy to use. Visit http://www.dovepress.com/testimonials.php to read real quotes from published authors. 